\title{
How long and what does it take for physical and emotional recovery after heart surgery?
}

\author{
Manuel J. Antunes, MD, PhD, DSc
}

\author{
From the Centre of Cardiothoracic Surgery, University Hospital and Faculty of Medicine of Coimbra, Coimbra, \\ Portugal. \\ Disclosures: Author has nothing to disclose with regard to commercial support. \\ Received for publication July 27, 2016; accepted for publication July 29, 2016; available ahead of print Aug 21, \\ 2016. \\ Address for reprints: Manuel J. Antunes, MD, PhD, DSc, Centro de Cirurgia Cardiotorácica, Hospitais da Uni- \\ versidade, 3000-075 Coimbra, Portugal (E-mail: antunes.cct.chuc@ sapo.pt). \\ J Thorac Cardiovasc Surg 2016;152:1557-8 \\ $0022-5223 / \$ 36.00$ \\ Copyright (c) 2016 by The American Association for Thoracic Surgery \\ http://dx.doi.org/10.1016/j.jtcvs.2016.07.052
}

Little is known about physical and mental recovery after open surgery. Because aortic valve replacement is currently one of the most frequent cardiac surgical procedures and a significant number of patients are of old age, this is a subject of significant interest to the surgical fraternity. In fact, we have come to learn that success of surgery is much more than just survival and absence of significant morbidity, and quality of life (QoL) after surgery is becoming a most important consideration.

In this issue of the Journal, Petersen and colleagues, ${ }^{1}$ from Hamburg, Germany, analyze the course of physical and mental recovery directly after conventional aortic valve surgery (AVS) in 60 patients followed up for 6 months, using several well-established measurement instruments applied preoperatively and sequentially at intervals of 4 weeks after AVS. They found that after a significant worsening of both parameters in the first week after surgery, there was a progressive recovery, the most pronounced seen within the first 6 weeks postoperatively. At 6 months, all parameters were significantly improved compared with preoperative values and significantly better or equal compared with published healthy norms.

Previous works have looked into physical and mental outcomes in the medium- and long-term after cardiac surgery, but I am not aware of such extensive and deep study of the weekly evolution of several physical and psychologic parameters immediately and in the short-term after AVS, which may influence our way of treating these patients. The current study involved a reasonable number of subjects for this kind of investigation and was well conducted, although less than half the patients had completed it. Naturally, the timing of the tests may have been influenced by individual levels of heart failure and anxiety, but it was constant throughout the study protocol and cohort, and the authors believe that its effect can be neglected.

The majority of patients involved in this study were discharged directly to their homes. Currently, there is a worldwide trend to send patients to rehabilitation institutions to speed up the recovery process. This is certainly often the be proven. ${ }^{2,3}$

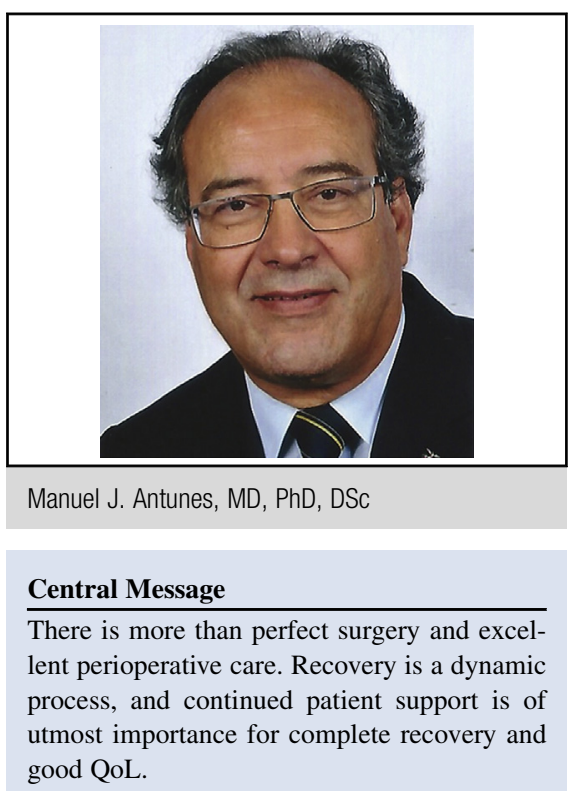

See Article page 1549.

case in the authors' country, but the advantages of the generalized use of this type of management over returning to one's own environment after cardiac surgery remains to

Of note, mental QoL improved immediately after surgery, whereas physical QoL took a little longer, reaching its peak at 6 months after surgery. This underscores the immensely important perioperative role of health professionals in stimulating the patients' psychologic and physical well-being. But because the recovery process continues at home, the family also has an essential role to play, and it is absolutely vital to recruit the next of kin while the patient is still in the hospital. At the moment of discharge, patients and their close family members must be clearly informed of what to do and how to do it. ${ }^{4}$ Support, encouragement, companionship, meaningful family activities, and conversation go a long way in speeding up emotional recovery and helping patients return to their highest levels of independent functioning.

In addition, community services, when available, also may be hired to assist in postdischarge management. Unfortunately, Petersen and colleagues ${ }^{1}$ did not stratify their patients by socioeconomic status (SES). Patients with low SES seem to be at greater risk for poor outcomes during the posthospital transition. Kangovi and colleagues ${ }^{5}$ found 
that patients with low SES are described discharge goals that are confusing and unrealistic in the face of significant socioeconomic constraints, and they hypothesized that "this goal misalignment leads to a cycle of low achievement and loss of self-efficacy that may underlie poor posthospital outcomes among low-SES patients." This is another factor that needs careful analysis and eventual correction both during hospital admission and at the time of hospital discharge.

Success means much more than just technically perfect surgery and excellent perioperative care. Postoperative recovery is a dynamic process, and continued posthospital support is of utmost importance if the main surgical goal of complete physical and mental recovery and good QoL is to be reached.

\section{References}

1. Petersen J, Vettorazzi E, Winter L, Schmied W, Kindermann I, Schäfers H-J. Physical and mental recovery after conventional aortic valve surgery. J Thorac Cardiovasc Surg. 2016;152:1549-56.e2.

2. Mampuya WM. Cardiac rehabilitation past, present and future: an overview. Cardiovasc Diagn Ther. 2012;2:38-49.

3. Laukkanen JA. Cardiac rehabilitation: why is it an underused therapy? Eur Heart J. 2015;36:1500-1.

4. Horwitz LI. Self-care after hospital discharge: knowledge is not enough. BMJ Qual Saf. 2016 [Epub ahead of print].

5. Kangovi S, Barg FK, Carter T, Levy K, Sellman J, Long JA, et al. Challenges faced by patients with low socioeconomic status during the post-hospital transition. J Gen Intern Med. 2014;29:283-9. 